\title{
Project 1640: the world's first ExAO coronagraphic hyperspectral imager for comparative planetary science
}

Ben R. Oppenheimer*a Charles Beichman ${ }^{\mathrm{b}}$, Douglas Brenner ${ }^{\mathrm{a}}$, Rick Burruss ${ }^{\mathrm{c}}$, Eric Cady ${ }^{\mathrm{c}}$, Justin Crepp $^{\mathrm{b}}$, Lynne Hillenbrand ${ }^{\mathrm{b}}$, Sasha Hinkley ${ }^{\mathrm{b}}$, E. Robert Ligon ${ }^{\mathrm{c}}$, Thomas Lockhart ${ }^{\mathrm{c}}$, Ian Parry ${ }^{\mathrm{d}}$, Laurent Pueyo ${ }^{\mathrm{e}}$, Emily Rice ${ }^{\mathrm{a}}$, Lewis C. Roberts, Jr. ${ }^{\mathrm{c}}$, Jennifer Roberts ${ }^{\mathrm{c}}$, Michael Shao ${ }^{\mathrm{c}}$, Anand Sivaramakrishnan ${ }^{\mathrm{f}}$, Remi Soummer ${ }^{\mathrm{f}}$, Gautam Vasisht ${ }^{\mathrm{c}}$, Fred Vescelus ${ }^{\mathrm{c}}$, J. Kent Wallace ${ }^{\mathrm{c}}$, Chengxing Zhaic, Neil Zimmerman ${ }^{\mathrm{g}}$

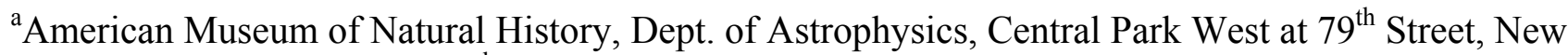
York, NY USA 10024-5192; ${ }^{\mathrm{b}}$ California Institute of Technology, 1201 California Blvd., Pasadena, CA USA 91125; ' Jet Propulsion Laboratory, California Institute of Technology, 4800 Oak Grove Dr., Pasadena, CA 91109, USA; ${ }^{\mathrm{d} I n s t i t u t e}$ of Astronomy, Cambridge University, Madlingly Road,

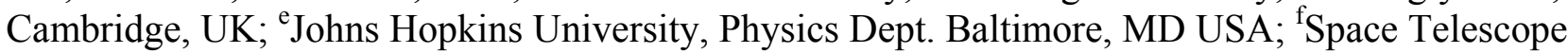
Science Institute, Baltimore, MD 21218, USA; ${ }^{\mathrm{g}}$ Max Planck Institute for Astronomy, Heidelberg, Germany

\begin{abstract}
Project 1640, a high-contrast spectral-imaging effort involving a coordinated set of instrumentation and software, built at AMNH, JPL, Cambridge and Caltech, has been commissioned and is fully operational. This novel suite of instrumentation includes a 3388+241-actuator adaptive optics system, an optimized apodized pupil Lyot coronagraph, an integral field spectrograph, and an interferometric calibration wave front sensor. Project 1640 is the first of its kind of instrumentation, designed to image and characterize planetary systems around nearby stars, employing a variety of techniques to break the speckle-noise barrier. It is operational roughly one year before any similar project, with the goal of reaching a contrast of $10^{-7}$ at 1 arcsecond separation. We describe the instrument, highlight recent results, and document on-sky performance at the start of a 3-year, 99-night survey at the Palomar 5-m Hale telescope.
\end{abstract}

Keywords: exoplanets, adaptive optics, coronagraphy, high-contrast imaging

\section{INTRODUCTION}

The history of science demonstrates again and again that technology drives scientific advances and can lead to the creation of entire subfields of inquiry. Exoplanetary science (the study of planets exterior to the solar system), which is nearly two decades old, started with the discovery of planets orbiting pulsars using extremely precise pulse time-ofarrival measurements. ${ }^{1}$ The field expanded rapidly with the invention of spectrometers capable of measuring the Doppler-shift of spectral lines with a precision of better than $3 \mathrm{~m} \mathrm{~s}^{-1} .2,3$ Since then, the discovery of transiting planets has permitted measurement of radii and even some spectroscopic data on the planets as they enter secondary eclipses. ${ }^{4}$ With the bright transiting systems such as HD $189733 \mathrm{~b}$ and HD209458b $\mathrm{b}^{5,6,7}$ among others, we have made the first steps in learning about the physical structure and composition of the atmospheres of highly irradiated, extremely hot planets. Finally, nine objects have been imaged so far that seem to be bona fide planets, though spectroscopic follow-up of these has been difficult. ${ }^{8}$ These measurements and associated survey work have transformed our view of the solar neighborhood. It is full of planets, and there is a real possibility that there may be more planets than stars in the Galaxy.

Observations of exoplanets unequivocally indicate tremendous diversity in planet characteristics, far beyond that imagined only ten years ago. ${ }^{9}$ We are on the verge of an incredibly rich, new, multidisciplinary field. The questions posed by comparative exoplanetary science are unlimited, but, to answer them requires a routine methodology to obtain spectra of exoplanets. Over the past fifteen years, we have developed the appropriate instrumentation to make such observations routine, as we demonstrate in this paper. With our third generation coronagraph and science camera, we

Adaptive Optics Systems III, edited by Brent L. Ellerbroek, Enrico Marchetti, Jean-Pierre Véran, Proc. of SPIE Vol. 8447, 844720 - (c) 2012 SPIE · CCC code: 0277-786/12/\$18 - doi: 10.1117/12.926419 
are embarking on a new survey of nearby stars to acquire near IR spectra of warm exoplanets in the mostly unexplored region from 5-50AU around nearby stars.

The system, called Project 1640, is fully functional and already achieving unprecedented contrast and sensitivity to a new region of parameter space.

\section{SPECKLES, OR WHY SUCH COMPLEX INSTRUMENTATION IS NEEDED}

The primary reason exoplanets are hard to see is our inability to adequately remove the starlight in the imaging system while leaving the reflected or emitted light from the planet unperturbed. This requires the manipulation of wave fronts from a neighboring solar system at the level of a nanometer to see objects up to 100 million times fainter than the star. Any defects in the optical path - whether from atmospheric perturbations of the wave front or from telescope and instrument optics-larger than this impose speckles in the final image plane that cannot be suppressed with the diffractive techniques of coronagraphy. Speckles are the spots that result from the constructive and destructive interference of wave front errors.

The importance of remnant starlight or "speckle noise" was not truly appreciated even just a few years ago, and now clever thinking and new data have driven the technology needed to conquer it. To understand this problem, one can use an example of one of the most precise coronagraphic images of a nearby star made to date. This is shown in Fig. 1, in which the star Alcor has been occulted by a coronagraph behind the 3388-actuator AO system on the 5-m Hale telescope. The figure shows three slices of one of our data cubes, at the wavelengths noted. Though $99 \%$ of the starlight has been removed, a broad and speckled pattern of light remains in the field of view (only 3.8"). These speckles are the primary source of noise obscuring exoplanets around stars.
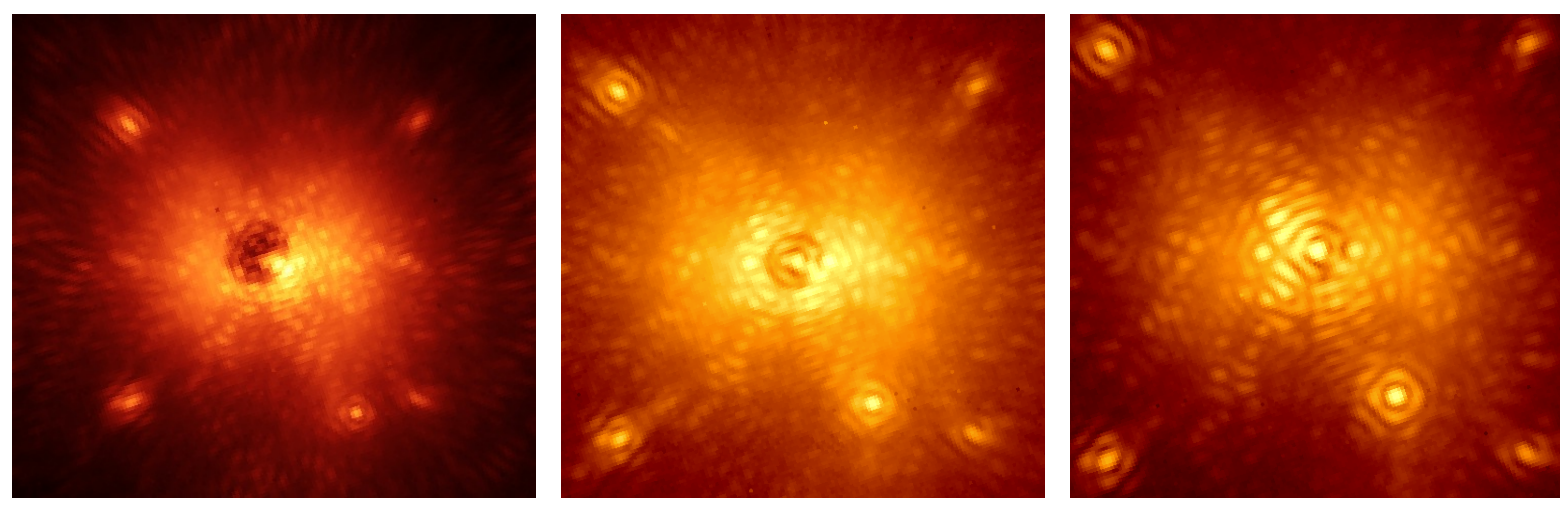

Figure 1. Three image slices at $\lambda=1120,1550$ and $1670 \mathrm{~nm}$ of the star Alcor with its M-dwarf companion (to lower right). Field of view shown is 3.0 arcsec. Note that the speckles, and four spots intentionally introduced to permit precision astrometry, change position as a function of wavelength, while the companion point source, located at the $\sim 5$ o'clock position, does not. This wavelength dependence permits discrimination between speckles and real celestial objects and is the primary way Project 1640 can go far below the speckle noise limit that has hampered previous instruments. We achieved $\sim 2$ mas relative astrometry between the star and companion, allowing us to confirm within weeks, that the companion orbits Alcor by using Alcor's known parallactic motion, rather than the more commonly used common proper motion. ${ }^{10}$

This source of noise can be many times larger than the photon shot noise at a given location in the image. ${ }^{11,12}$ It is highly correlated, meaning that simply integrating longer does not improve sensitivity. ${ }^{13}$ The speckles that remain in an image of this nature are not due to atmospheric wave front distortions. The AO system controls those, and the remainder dynamically average to a smooth halo. These residual speckles are long-lived, and we have observed some to last for well over an hour. ${ }^{13}$ They are due to small wave front errors $(<100 \mathrm{~nm}$, or about $\lambda / 15$ at $\mathrm{H}$ Band $)$ imposed by the optics between the AO system and the science detector. These are called "non-common path" errors because the optics are not in the part of the beam that is sensed by the AO system's wave front sensor. A number of authors have pointed out that these "quasi-static" speckles can be "tamed" by taking images at multiple wavelengths over each pass band. ${ }^{14-16}$ This was the technical motivation for building the P1640 Integrated Field Spectrograph (IFU), shown in Fig. 6. 
To understand the gain in sensitivity provided by integral field data consider the different behavior of a speckle as compared to a true point source as a function of wavelength. In Fig. 1, the companion star is clearly seen in this sequence of images always at the same location on the sky. The speckles, in contrast, change position radially as the wavelength changes. The speckle size $(\sim \lambda / D)$, comparable to the PSF, changes, but so does the location of each speckle. Thus the speckles trace diagonal loci through the data cube, while the companion traces a path with constant $\mathrm{x}, \mathrm{y}$ location.

The fact that speckles behave so differently from real sources in these three-dimensional data cubes ( $\mathrm{x}, \mathrm{y}, \lambda$ coordinates) means that one can discriminate real point sources from speckles through statistical analysis and three-dimensional model fitting of the data. The human eye, for example, is extremely good at picking up patterns such as these, and the data in Fig. 1 can also be seen in movie form while stepping through frames at separate wavelengths. The steady companion is easily distinguished. Since we can now measure this effect, we can control it and get past the speckle noise. This has the potential to yield two or more orders of magnitude in sensitivity ( 6 magnitudes; see below), meaning that Jupiter-mass companions will be detectable by the Project 1640 coronagraph around $\sim 200$ Myr old stars in our survey $(\S 4)$. Furthermore, the data provide low-resolution spectra of the faint companions, the key to comparative exoplanetary science.
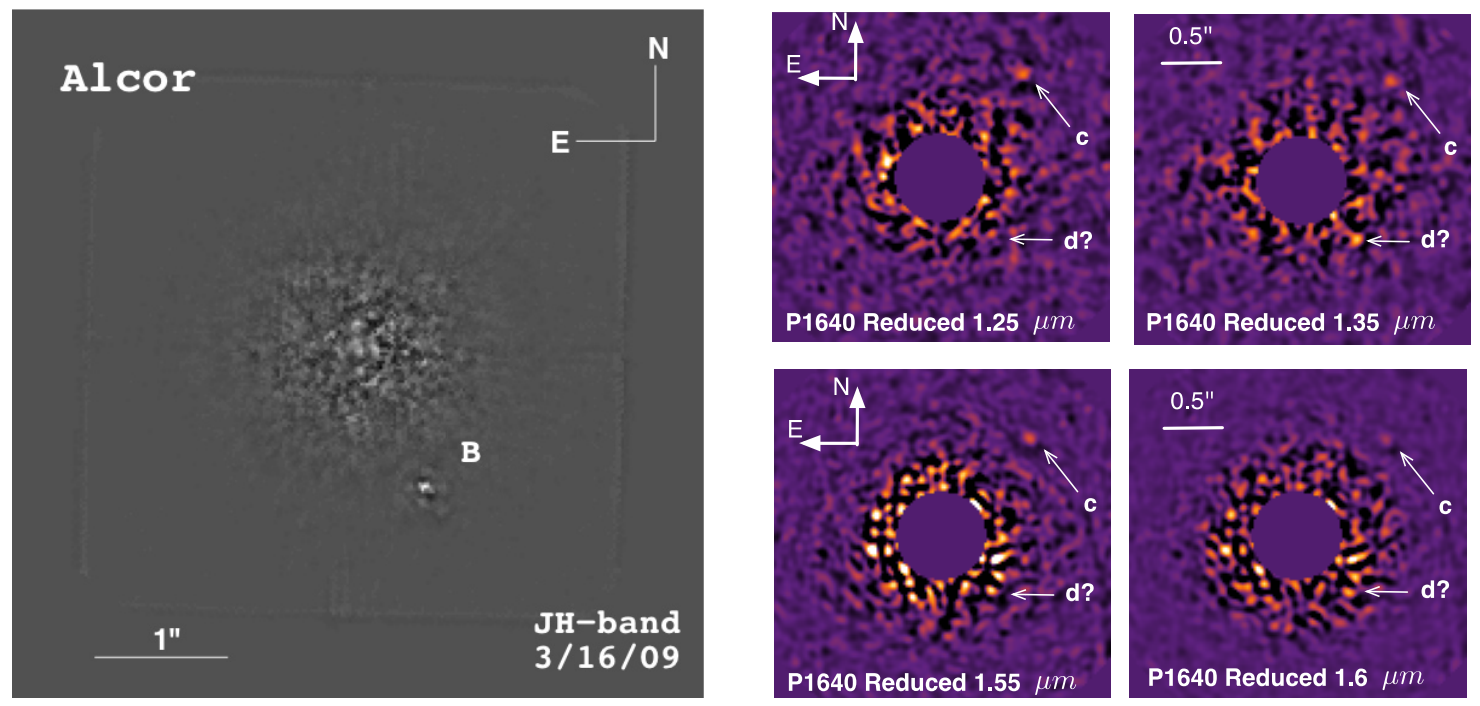

Figure 2. (left) The Alcor data cube after a modified LOCI speckle suppression algorithm has been applied to the data. ${ }^{17}$ Compared to Fig. 1, Alcor B is detected here with 64 times higher SNR. (Right) 4 separate spectral images (data cube slices) of planets HR 8799 "c" and "d" (HR 8799b is outside the field of view) acquired in Dec. 2011, with speckles suppressed using another method. ${ }^{18}$ The spectroscopic detection of " $\mathrm{c}$ " is robust, but " $\mathrm{d}$ " is marginal due to poor seeing (1.6-2.0 arcsec) which leads to diminished AO and coronagraphic performance.

We have already implemented two types of innovative speckle reduction software, and achieved a gain of 3 to 4 magnitudes of sensitivity penetrating through the speckle noise. These reduction techniques are summarized in two papers. ${ }^{17,18}$ We have spent considerable effort not only to improve the SNR of faint objects but also to derive their spectra from the speckle-reduced images. An example of the speckle reduction is shown in Fig. 2, where Alcor's companion is now seen with an increase in SNR of a factor of 64 after speckle suppression. We note that other speckle removal techniques that obtain diversity in measurement of speckles have been successful in cases such as the direct imaging of the HR 8799 planetary system. ${ }^{19}$ These techniques usually employ field rotations rather than chromatic speckle movements. Another technique involves polarimetry. It effects the only known complete speckle removal at the $10^{-8}$ level, but requires that the object of interest is polarized. ${ }^{20}$

It should be noted that a companion closer and fainter to the parent star than Alcor's, in the data shown here, would become lost in the sea of speckles, and therefore not visible to the naked eye by viewing movies. This is where the power of the speckle reduction software will have a major impact. 
But this software subtraction of speckles only removes the structured light in the image that obscures true sources. The photon noise associated with the speckles remains a fundamental noise source. For this reason we are dramatically advancing the field of high contrast imaging even further by working to eliminate the speckles and their associated noise optically, before the science detector, using advanced $\mathrm{AO}$, optimized coronagraphy and a new type of wave front sensing.

\section{INSTRUMENTATION}

Project 1640 includes four optical components mounted on the Palomar 5-m Hale Telescope: a 3388+241-actuator Adaptive Optics (AO) system, ${ }^{21,} 22$ a diffraction-limited, apodized-pupil Lyot coronagraph ${ }^{23}$ (APLC), a diffractionlimited integral field spectrograph ${ }^{24}$ (IFU), and finally a calibration wave front $\operatorname{sensor}^{25,26}$ (CAL). In addition, we have two software "components," an automated data pipeline to extract calibrated data cubes from each IFS exposure ${ }^{27}$; and an automated speckle suppression $\operatorname{code}^{17}$ with optimized spectral extraction. ${ }^{18}$

Since we have published the details of these techniques and instruments elsewhere, ${ }^{24}$ in this paper we simply summarize the instrumentation and describe our survey, current performance and initial results from commissioning observing time. Table 1 shows the current operating parameters for the IFU, coronagraph and AO system.

Table 1. Principal instrument properties

\begin{tabular}{|c|c|}
\hline \multicolumn{2}{|c|}{ Project 1640 IFU + Coronagraph } \\
\hline Wavelength coverage & $\begin{array}{c}0.98-1.75 \mu \mathrm{m}, \\
\Delta \lambda=0.77 \mu \mathrm{m}\end{array}$ \\
\hline Detector & Teledyne H2RG \\
\hline IFU FOV & 3870 mas \\
\hline Plate scale & $19.35 \mathrm{mas} / \mathrm{lenslet}$ \\
\hline Number of spectra & $200 \times 200=40,000$ \\
\hline Pixels per spectrum & $3.2768 \times 32$ \\
\hline$\Delta \lambda$ per pixel & $.025 \mu \mathrm{m}$ \\
\hline $\mathrm{R}=\lambda / \Delta \lambda$ & 39 to 70 \\
\hline Lenslet Pitch & $75 \mu \mathrm{m}$ \\
\hline $\begin{array}{l}\text { Input f/ratio from coronagraph } \\
\text { for } \lambda / 2 \mathrm{D} \text { Spaxels at } 1.0 \mu \mathrm{m}\end{array}$ & $f=143.21$ \\
\hline Focal Plane Mask size & $5.6 \lambda / \mathrm{D}$ \\
\hline $\begin{array}{l}\text { Optimal coronagraph } \\
\text { wavelength }\end{array}$ & $1.640 \mu \mathrm{m}$ \\
\hline Apodizer throughput & $51 \%$ \\
\hline Total System Throughput & $12.3 \%$ \\
\hline \multicolumn{2}{|c|}{ Palomar 3000 Actuator AO System } \\
\hline Telescope Diameter & $\mathrm{D}=5.05 \mathrm{~m}$ \\
\hline$\theta_{\mathrm{AO}}=\mathrm{N}_{\mathrm{act}} \lambda / 2 \mathrm{D}(1-1.8 \mu \mathrm{m})$ & 1010 to 1818 mas \\
\hline$\lambda / 2 \mathrm{D}$ at $0.98 \mu \mathrm{m}$ & $20.04 \mathrm{mas}$ \\
\hline Current Output f/ratio & 15.4 \\
\hline
\end{tabular}

\subsection{Coronagraph design}

To determine the best combination of mask parameters for the APLC using the techniques in Soummer et al. ${ }^{23}$, we have optimized our mask characteristics by calculating the expected contrast and PSFs for the system. Considering just the Hband alone, ignoring the J-band, we identified a very good solution for the H-band which is also acceptable at J. We have found that although optimum solutions for the combination of $\mathrm{J}$ and $\mathrm{H}$ bands exist, they require masks that are much larger, impacting the inner working angle (IWA) too much. In addition, the AO system will not deliver the same performance at $\mathrm{J}$ band, so it is not necessary to have the same theoretical contrast at $\mathrm{J}$ band. However, we find that we can improve the performance at $\mathrm{J}$ band by oversizing the Lyot stop central obstruction to eliminate most of the J-band 
leakage in the Lyot plane. Putting these two issues together, we re-optimized the H-band solution at the same time and find a new solution with the Lyot mask slightly larger. The parameters of the optimized H-band solution are not affected by the Lyot stop oversizing, so that the J-band performance can be improved by a factor of a few by oversizing the Lyot stop.

Fig. 3 shows the theoretical contrast in the absence of wave front errors as a function of central wavelength and mask size. Fig. 4 shows the contrast for the chosen design as a function of radial distance from the occulted star.

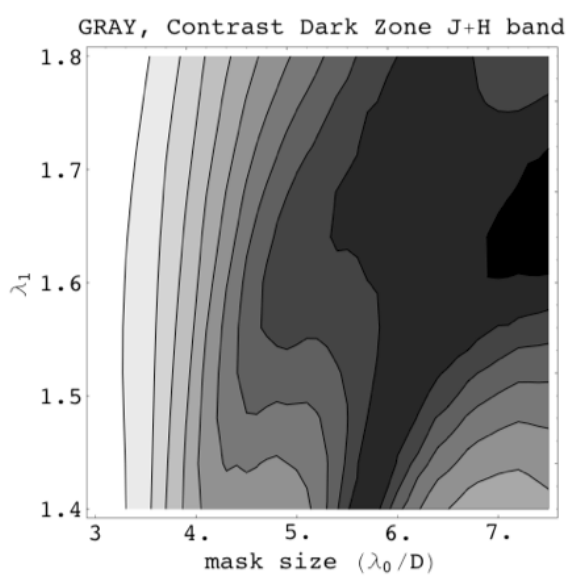

Figure 3. Parameter space for the two main parameters. Abscissa: mask size at band center lambda $=1.64 \mu \mathrm{m}$. The vertical axis corresponds to the wavelength at which the prolate apodizer is applied. Grey scale is a unit-less metric indicating contrast, with black being the highest contrast. ${ }^{23}$

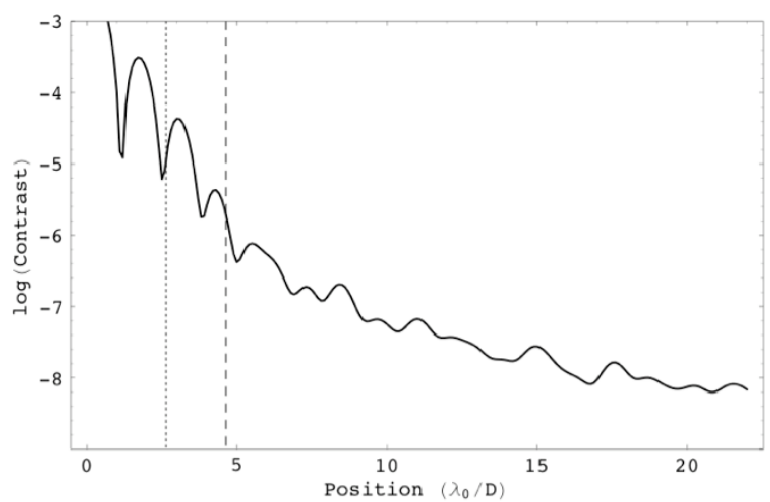

Figure 4. H-band contrast without aberrations. The dotted line is the focal plane mask radius, and the dashed line is a conservative inner working angle (radius of the mask + two resolution elements).

The coronagraphic masks - apodizer, which is a microdot etched function on glass; focal plane mask, which is a gold mirror with a precision etched hole in the center; and the Lyot stop, wire-EDM cut aluminum—are all shown in Fig. 5.

\subsection{Adaptive Optics and Calibration System}

The high-order Palomar adaptive optics system operates with a 3388-actuator deformable mirror (DM) with $500 \mathrm{~nm}$ stroke updating at roughly $2 \mathrm{kHz}$, along with a lower-order 241 -actuator DM with far greater stroke $(5 \mu \mathrm{m})$. This socalled P3k system is operational as of summer 2011, and we had our first commissioning runs with it in December 2011 and June 2012. The purpose of such a high-density of actuators is to be able to control the wave front with more degrees of freedom such that the inner region of the image is cleared of speckles, cleaning out the so-called AO control region. ${ }^{28}$ This requires that the wave front be sensed at the location in the optics where it is needed to be optimal - at the science camera (IFU). However, between those optics and the P3k wave front sensor there are 11 other optical surfaces, which form the heart of the coronagraph itself. Without being able to sense the wave front imperfections induced by these 
optics, the additional actuators do nothing but correct the atmosphere better. P3K reduces the seeing halo of starlight, but without additional information cannot suppress the quasi-static speckles.
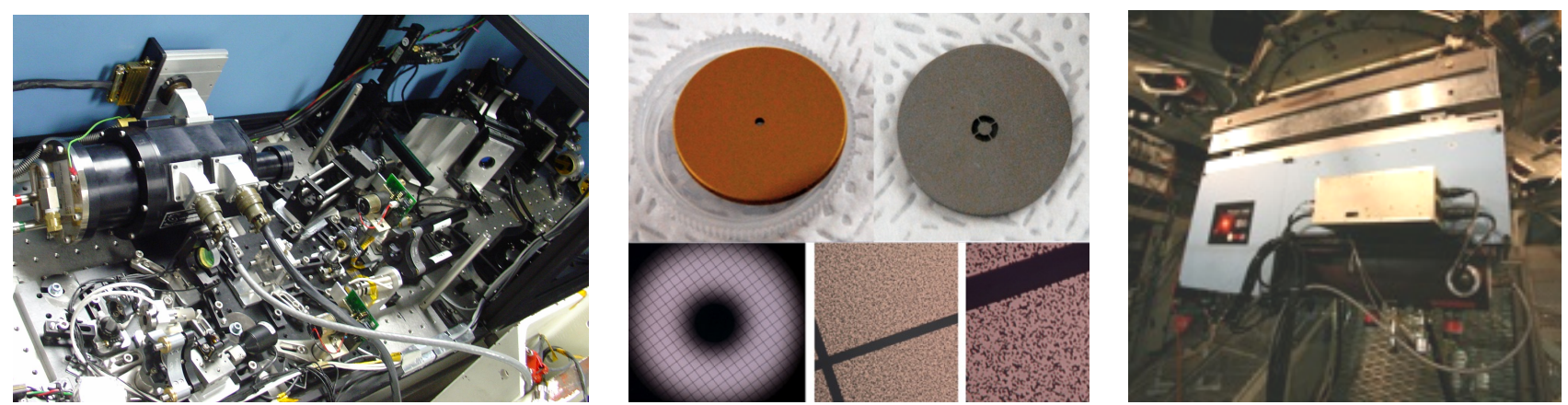

Figure 5. From left to right: the CAL System, with coronagraph at right; coronagraph optics (middle; including focal plane mask, Lyot stop and apodizer); and the fully assembled suite of instruments mounted on the P3k optical bench at the Cassegrain focus of the Palomar 200-inch telescope. The coronagraph and CAL system are contained within the blue enclosure, while the IFU dewar hangs below on the right. There are 6 electronics racks, 23 computers, two deformable mirrors (241 and 3288 actuators), two tip/tilt systems, two IR cameras, 28 motors, and a fast wave front sensor used in the operation of this instrument. The concept of this system is similar to that of GPI and the VLT-SPHERE projects, but P1640 is operational roughly one year earlier.

Thus, a $4^{\text {th }}$ component has been built by NASA-JPL, called the CAL system, short for calibration system (Fig. 5). CAL consists of a small Mach-Zehnder interferometer that measures the wave front aberrations by interfering the light of the star that is rejected by the coronagraph (the point-source core of the PSF) and $20 \%$ of the light that is otherwise due to enter the IFS. This allows measurement of the wave front right before the light enters the IFS science camera. Because the measurement is interferometric in nature, we are able to determine the full electric field of the incoming light and can thus also correct for wave front amplitude variations. The goal is to measure at a rate of roughly $1 \mathrm{~Hz}$, much faster than the quasi-static speckle lifetime, to allow for very high SNR wave front determination. The reconstructed wave front is then fed back to the $\mathrm{AO}$ system to drive the $\mathrm{AO}$ to an optimal wave front within the coronagraph, immediately prior to the science camera. The result is to cut a dark hole (see Fig. 7) in the speckle pattern that has a minimum radius of $32 \lambda / \mathrm{D}$ - the P1640 field of view at its longest wavelength. The CAL system is designed to measure wave fronts with a precision of $1 \mathrm{~nm}$, which will result in a speckle floor within this hole that is about $10^{-6}$ to $10^{-7}$ times fainter than the star. Coupled with the software speckle suppression it is our goal to achieve sensitivity to objects at the $10^{-7}$ contrast level or better.

This CAL system has been built and installed (Fig. 5). We have achieved on-sky wave front measurements starting in December 2011, and can achieve control of the higher order wave front to $\sim 5-10 \mathrm{~nm}$ rms. A CAL generated $32 \lambda / \mathrm{D}$ dark hole is shown in Fig. 7.

\subsection{IFU}

The IFU is described in detail elsewhere, ${ }^{24}$ but we summarize the optical design here. This IFU is a lenslet-based system in which the science focal plane is imaged on a $75 \mu \mathrm{m}$-pitch 200x200 lenslet array. The lenslet array forms tiny pupil images which are then dispersed by a prism. Each lenslet, thus, is a spatial pixel, and the microspectra from all of the lenslets are imaged on the H2RG detector. The spectra are closely packed, but can be extracted with cross-talk on the level of less than a few percent. The optical design is compact, primarily because of space constraints imposed by the P3k AO system and the allowable instrument volume. The IFU is operated with $\mathrm{LN}_{2}$ with a hold time of about 36 hours. The detector is maintained at $80 \mathrm{~K}$ and read out using Teledyne's ASIC and JADE2 system. Teledyne's IDL data acquisition software is used in the field reliably. This greatly minimized software development and implementation time. Fig. 6 shows the cryogenic optics and layout before baffling was inserted, in the clean room at AMNH. 


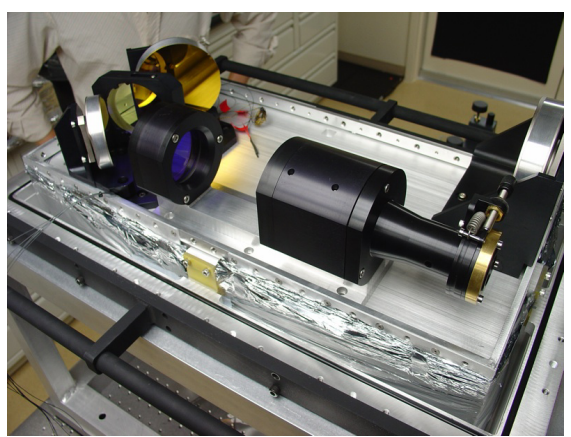

Figure 6. Cryogenic optics of the integral field spectrograph which serves as the science camera for Project 1640 . The sensor is a Teledyne H2RG detector. The system is shown open in the AMNH lab prior to deployment. This instrument produces $\sim 40,000$ spectra from 0.98 to $1.75 \mu \mathrm{m}$ over a field of view of 3.87 arcsec square. A complex data pipeline converts this into cubes of RA, $\delta$, and $\lambda$.

\section{THE SURVEY}

\subsection{Sensitivity}

In designing an exoplanet imaging survey two quantities are particularly important for picking the optimal set of stars to observe: the system's blank-sky limiting magnitude over a one hour observation, and the contrast that the instrumentation can deliver as a function of linear separation from the star.

The limiting magnitude sensitivity of our instrument is $\mathrm{H}=21.5^{\mathrm{m}}$ in one hour, for a $5-\sigma$ detection in a single slice of the data cube (with bandwidth of $\Delta \lambda=25 \mathrm{~nm}$ ). This number determines the faintest object that is detectable in any image, in the total absence of primary star light which produces both a seeing halo and a speckle background.
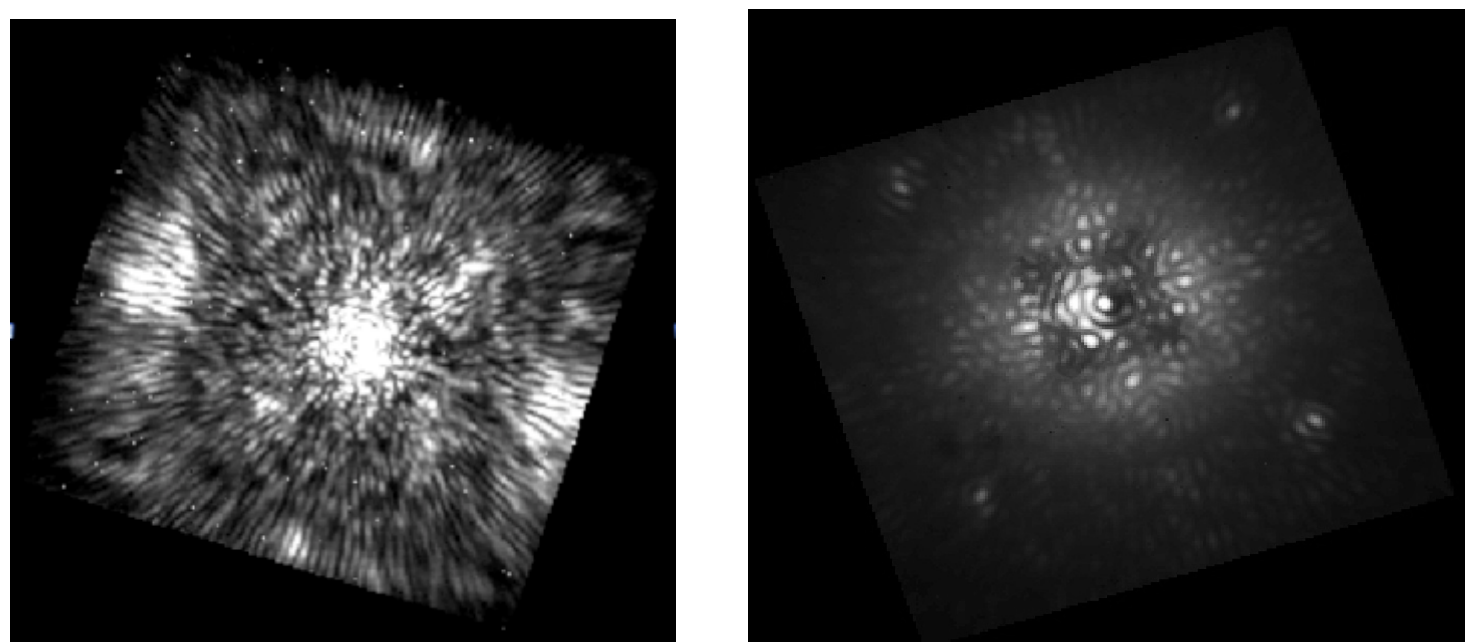

Figure 7. (left) A J band data cube slice showing the formation of a $32 \lambda / \mathrm{D}$ square dark hole after correcting non-common path phases over $\sim 10$ CAL iterations. The atmospheric halo has been filtered out in this image. (right) Image of a slice of a data cube for a star observed with the new PALM 3000 AO (CAL is turned off), in October 2011. Note that the AO system has begun to carve out a darker "hole" in the PSF in the immediate vicinity of the star. For best contrasts, both AO and CAL work in real time to mitigate atmospheric and instrumental speckles, respectively.

To understand the contrast achievable, we have (1) measured the contrast with the full system in operation without CAL correction, (2) measured it again with CAL correction in the lab in March 2012, and (3) we have conducted extensive simulations of the entire optical system grounded in the performance metrics we have already measured in both Phase I and the first observing runs with the revamped system in operation. The result is that without the CAL system in operation we achieve a contrast before speckle suppression of $10^{-5}$ at 0.5 " radius from the star. This number is relatively 
constant through the dark hole region that the AO system can control (radius of $32 \lambda / \mathrm{D}$; see Fig. 7). With CAL in operation, this number drops to about $8 \times 10^{-7}$ in the worst case scenario $\left(\mathrm{H}=8^{\mathrm{m}}\right.$, slightly worse than average seeing at Palomar). Combining the proven speckle suppression improvement of at least a factor of 60 (to be conservative), the survey will operate at the $10^{-7}$ contrast level or better, just outside the coronagraph mask. In Fig. 7 we show an image from the October 2011 observing run in which we detected the dark hole that the AO system is capable of producing.

These various metrics are folded into a simulation based on an actual star list in order to translate them into sensitivity to companions as a function of mass and orbital separation. ${ }^{29,30}$ Sample selection is discussed in the next section. Note that the sensitivity of the survey is different for every star in the mass-separation parameter space, due to varying ages, and distances of the stars. Fig. 8 shows the composite sensitivity to companions between 2 and 40AU, for a subset of stars at an age of $200 \mathrm{Myr}$ and various distances (the reason for the fall-off in sensitivity to larger semi-major axis because these fall out of the field of view for the closer stars). The survey clearly is sensitive through a large fraction of the gas-giant planet regime.

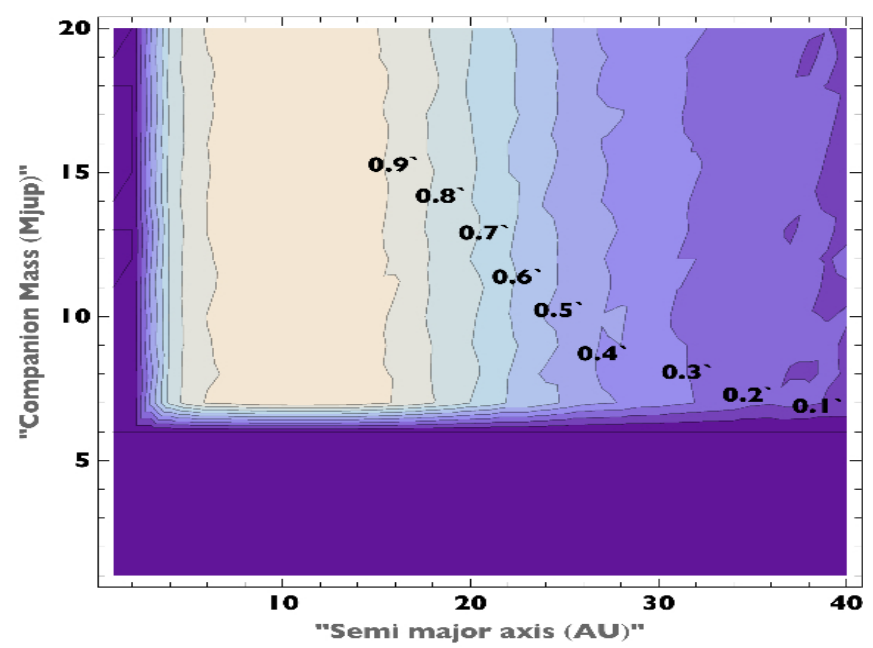

Figure 8. Discovery space around a subset of typical (200 Myr old) stars for the survey, as described in the text. The numbers and corresponding regions indicate the fraction of objects in that part of parameter space that will be detected if they actually exist. ${ }^{30}$

\subsection{Star Selection: The A0V-F5V Survey}

Stars with spectral types between A0 and F5 offer a number of advantages for a coronagraphic survey. Most importantly, radial velocity (RV) and transit studies indicate that the incidence of giant planets increases in proportion to stellar mass with as many as $14 \%$ of A stars having a $>1 \mathrm{M}_{\mathrm{J}}$ companion ( $\left.<5 \mathrm{AU}\right)$ compared with only $2 \%$ of M stars. ${ }^{31}$ Theoretical considerations preferentially favor A star hosts for the formation of giant planets on wide orbits via disk fragmentation. ${ }^{32}$ These theoretical and observational concepts appear to be borne out by recent imaging detections of giant planets orbiting nearby A stars, HR $8799^{19,33}$ and $\beta$ Pic. ${ }^{34}$ Finally, the intrinsic brightness of A and F stars makes them suitable for AO imaging while their youth (many $<1$ Gyr) means that their companions are bright enough for detection. $^{35}$

We have selected our sample according to the following criteria: spectral type A0-F5 with luminosity classes IV and V; distances $<50 \mathrm{pc}$ for $\mathrm{F}$ stars and $<75 \mathrm{pc}$ for A stars; declination between $-20^{\circ}$ and $+70^{\circ}$; no companions within 0.1 " to 4"; brightness limits of $\mathrm{H}<7^{\mathrm{m}}$ and $\mathrm{V}<8^{\mathrm{m}}$. Metallicity and ages are critical parameters with the incidence of planets increasing as $\left.\sim 10^{1.2[\mathrm{Fe} / \mathrm{H}]}\right]^{31}$ and the brightness of planets being a steeply increasing function of decreasing age. We have taken ages from the literature based on cluster membership or isochrone matching. ${ }^{36-40} 25 \mu \mathrm{m}$ excess emission can also be a signpost for ages $<400 \mathrm{Myr}$ among $\mathrm{A} \mathrm{stars}{ }^{41}$ and we have incorporated IR data from Spitzer and the recently released WISE catalog into our prioritization.

Our sample includes approximately 200 A and F stars (102 A0-A4, 32 A5-A9, and 66 F0-F5 stars) with a distribution in distance, age and spectral type as shown in Fig. 9. We have prioritized our targets and will ultimately interpret our results using Monte Carlo simulations that incorporate planetary and host star properties and P1640 instrumental 
characteristics to predict planet yield under various assumptions about the incidence of planets of a given mass and their orbital location. ${ }^{24,42,43}$ In addition to the physical characterization of individual planets, our primary survey results will be constraints on the distributions of planetary orbits and estimates of the fractional incidence of planets as a function of planet and host star mass.

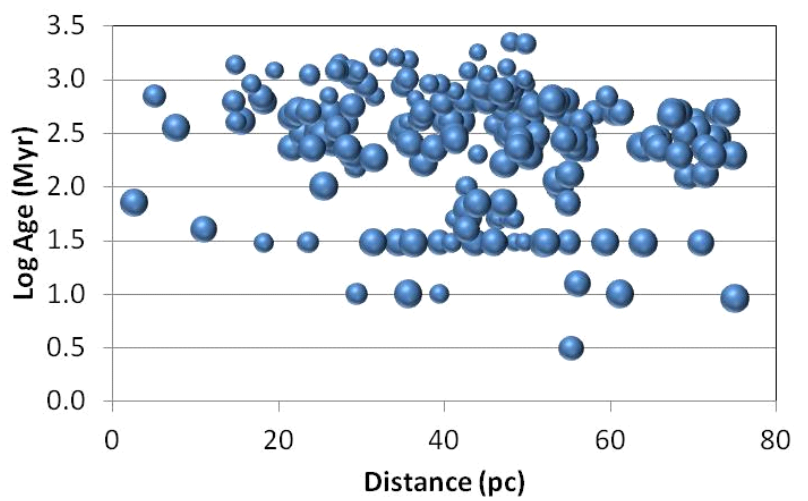

Figure 9. Distribution of 200 target stars as a function of age, distance and spectral type with large symbols representing A stars and the smallest symbols are early F stars.

Assuming a limiting $10^{-7}$ contrast after speckle reduction (which is conservative) and $\mathrm{H}<21.5 \mathrm{mag}(5-\sigma)$ limiting magnitude for spectral data, the detection probability averaged over 200 stars is $5-10 \%$. Integrating over the probability of detecting a planet around each star, we estimate that we will detect between 10-20 planets with an average mass of 9 $\mathrm{M}_{\mathrm{J}}$ and SMA $=60 \mathrm{AU}$ (Fig. 10). The detection probability drops by a factor of 2-5 with a factor of 10 decrease in contrast and by $25 \%$ with a one magnitude decrease in limiting magnitude to $\mathrm{H}<20.5$ mag. This survey will also characterize the so-called "brown dwarf desert" and achieve low resolution spectra of at least several planet-mass objects. We will examine 5-8 objects per night. A Monte Carlo simulation of our observing strategy incorporating our target list, the length of any given night, $30 \%$ favorable weather conditions, return visits to $10 \%$ of the targets for followup and confirmation, and reasonable overheads indicates that a 200 star survey is a reasonable match to the 99 nights in the survey. The yield for the survey is represented in Fig. 10.

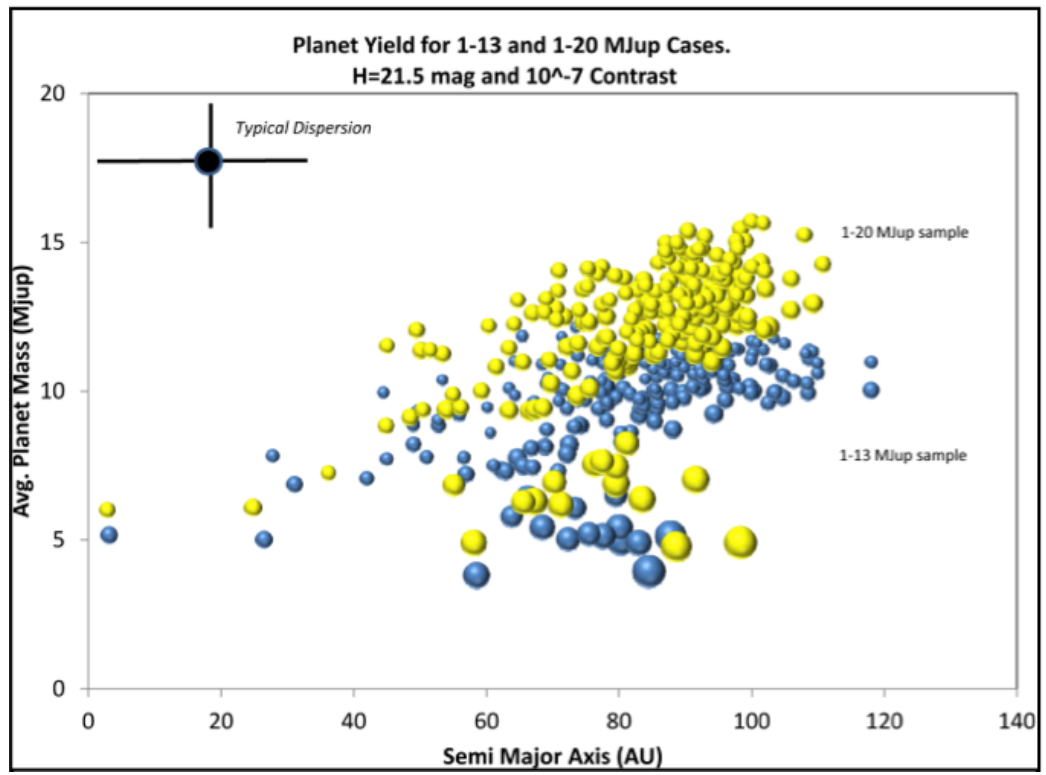

Figure 10. Expected planetary properties on a per star basis from a Monte Carlo simulation of 220 stars for a survey with $10^{-7}$ contrast at $1 "$ and a $\mathrm{H}<21.5 \mathrm{mag}$ limit. The size of the symbol is proportional to probability of detecting a planet, with the largest symbol being roughly $60 \%$ and the smallest about $10 \%$. In total we expect to find 10-20 planets from our sample. The yellow (light color) and blue symbols represent different upper limits of the "planet" mass range assumed in the simulation. 


\subsection{Companion Characterization}

On another front, we have been working on a complex set of analysis tools that rely on Monte Carlo modeling and hundreds of input model and observed spectra to determine the surface gravity and temperature of companions found. We have demonstrated that even with our low-resolution spectra (32 wavelength channels across the 0.98-1.75 m bandpass), distinguishing molecular features and spectral types is entirely practical, particularly toward lower temperatures. The software fits each of the $\mathrm{J}$ and $\mathrm{H}$ bands separately and in a second step simultaneously to find the best fit solution. Fig. 11 shows comparisons of input type-specimen spectra from M1 to T4.5 overlaid with the low resolution spectra that P1640 produces. For example, we have applied this technique applied to the early M-dwarf companion to HD 129333. ${ }^{44}$ With these models and the search algorithm, a solution for each object can be found relatively quickly. We are currently expanding the algorithm to include other parameters in the search space, such as metallicity.
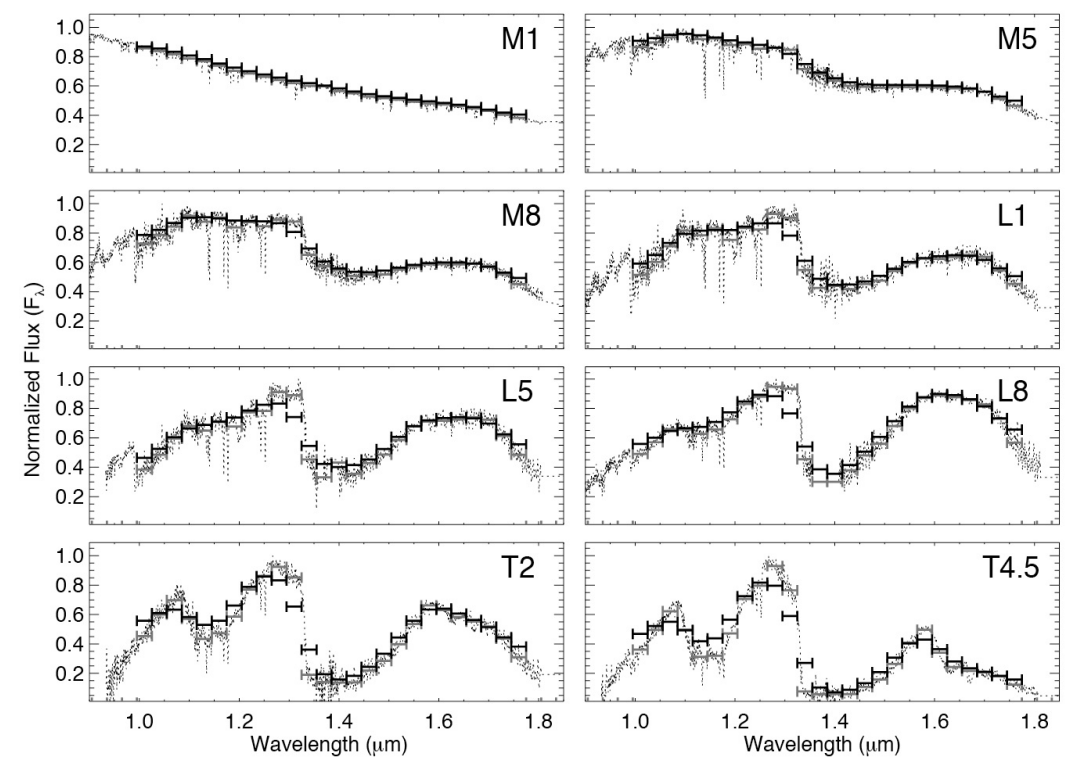

Figure 11. Demonstration of the sufficiency of the P1640 spectrograph resolution for determining spectral type. A set of tools to arrive at a multi-dimensional best fit based on input models and observed spectra from other projects permits determination of effective temperature and surface gravity, with metallicity to be added into the software soon.

\section{RESULTS AND FUTURE WORK}

Current on-sky performance has not reached full potential of the system regularly, with additional engineering during the summer of 2012. In June 2012, we demonstrated that the system will reach its designed performance levels. Furthermore, the detection and acquisition of the spectrum of HR 8799c (Fig. 2) under bad seeing conditions in December 2011 clearly shows that we are operating in new high-contrast parameter space. The contrast achieved in the lab is shown in Fig. 12. On sky contrast is roughly a factor of 10 worse. These contrasts are the 5- $\sigma$ raw contrasts computed using the methodology in Hinkley et al. ${ }^{13}$ Because the system has significant amplitude variations (which appear to originate in the optics before the coronagraph), simple phase-only correction and wave front sensing by the CAL system (and fed back to the AO control loop) does not achieve the contrast needed for the survey to be most effective. However, the CAL system actually senses the full electric field of the light in the coronagraph. Thus, both amplitude and phase variations can be controlled. We have implemented two different algorithms to do this and create half-plane dark holes in the coronagraphic PSF. If this ends up being the primary mode of operation for the survey, the search portion of the observing will require more observing time for each star (though follow-up will not require that). We show the results of the half-plane dark hole and associated contrast curves in Fig. 12. 

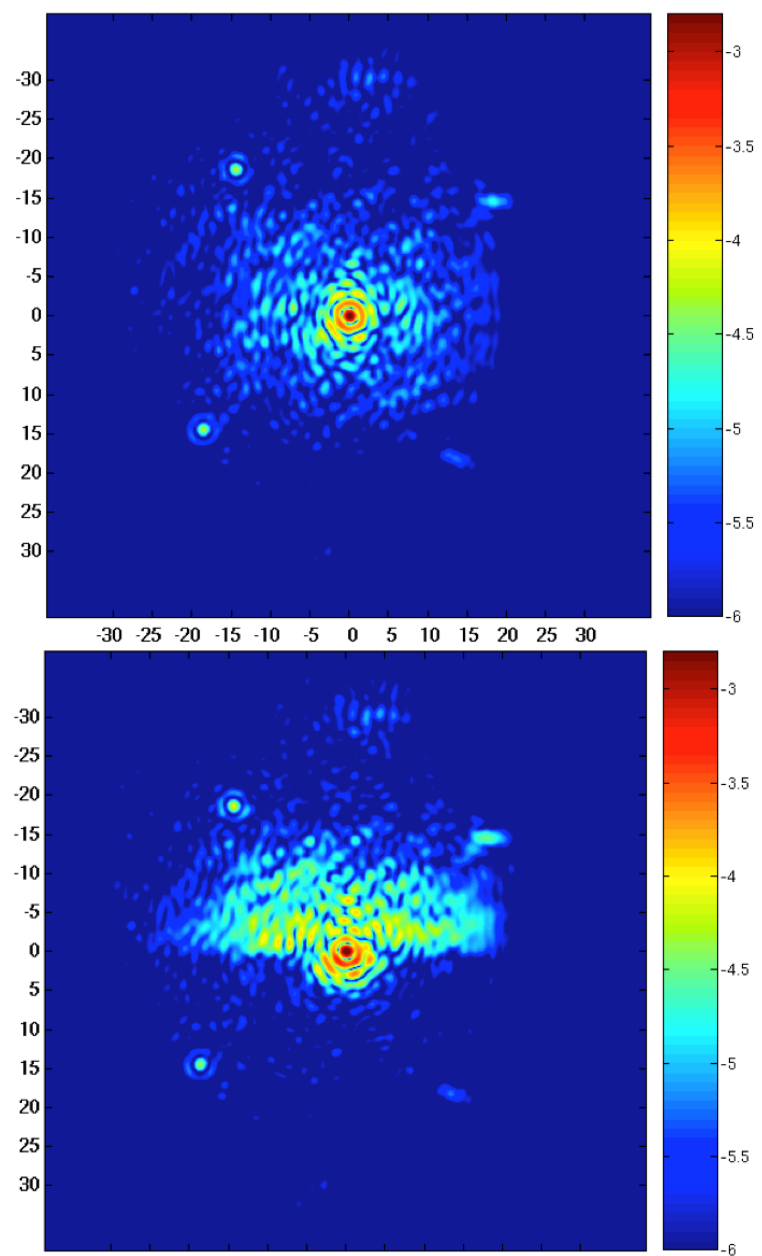
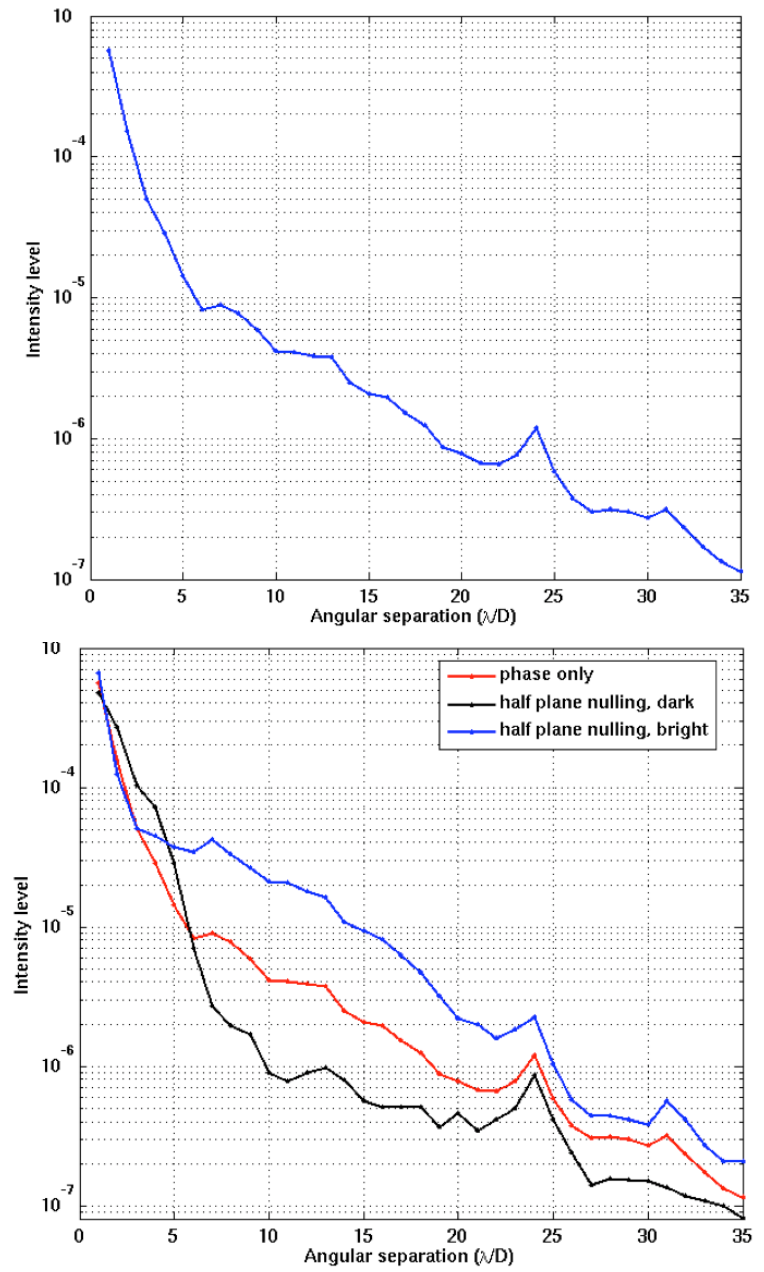

Figure 12. In lab PSFs and contrast curves. At top, image on left and contrast on right, with full correction in operation with CAL controlling only the phase of the starlight. Bottom: 15 iterations of one of the half-plane dark hole techniques. In this case, the raw contrast reaches below $10^{-6}$ at $10 \lambda / \mathrm{D}$. Speckle suppression will take the actual contrast to the project's goal $\left(10^{-7}\right)$ or better at all radii outside the coronagraphic mask.

\section{REFERENCES}

[1] Wolszczan, A., Frail, D.A., “A planetary system around the millisecond pulsar PSR1257+12,” Nature, 355, 145-147 (1992).

[2] Marcy, G.W., and Butler, R.P., "Planets Orbiting Other Suns," Pub. Astron. Soc. Pac., 112, 137-155 (2000).

[3] Mayor, M. and Queloz, D., "The Mass Function Below the Substellar Limit," ASP Conf. Series, 154, 77-81 (1997).

[4] Charbonneau, D., Allen, L. E., Megeath, S.T., Torres, G., Alonso, R., Brown, T., Gilliland R. L., Latham, D. W., Mandushev, G., O'Donovan F. T., and Sozzetti, A., "Detection of Thermal Emmision from an Extrasolar Planet," Astrophys J. , 626, 523-529 (2005).

[5] Richardson, L. J., Deming, D., Horning, K., Seager, S.,\& Harrington, J., "A Spectrum of an Extrasolar planet," Nature, 445, 892-894 (2007).

[6] Grillmair, C. J., Charbonneau, D., Burrows, A. et al., "A Spitzer Spectrum of the Exoplanet HD 189733b," Astrophys. J., 658, L115-L119 (2007).

[7] Swain, M. R., Tinetti, G., Vasisht, G. et al., "Water, Methane, and Carbon Dioxide Present in the Dayside Spectrum of the Exoplanet HD 209458b," Astrophys. J., 704, 1616-1621 (2009). 
[8] Traub, W.A., and Oppenheimer, B.R., 2010, "Direct Imaging of Exoplanets," [Exoplanets], University of Arizona Press, Phoenix, 111-156 (2010).

[9] Oppenheimer, B.R. and Hinkley, S., "High Contrast Observations in Optical and Near-IR Astronomy," Ann. Rev. Astron. Astrop., 49, 253-289 (2009).

[10] Zimmerman, N., Oppenheimer, B.R., Hinkley, et al., "Parallactic Motion for Companion Discovery: An MDwarf Orbiting Alcor," Astrophys. J., 709, 733-740 (2010).

[11] Labeyrie, A., "Images of exo-planets obtainable from dark speckles in adaptive telescopes," Astron. Astrophys., 298, 544-552 (1995).

[12] Marois, C., Doyon, R., Racine, R., Nadeau, D., 2000, "Efficient Speckle Noise Attenuation in Faint Companion Imaging," Pub. Astron. Soc. Pac., 112, 91-97 (2000).

[13] Hinkley, S., Oppenheimer, B. R., Soummer, R., Sivaramakrishnan, A., Roberts, L. C., Kuhn, J., Makidon, R. B., Perrin, M. D., Lloyd, J. P., Kratter, K., and Brenner, D., "Temporal Evolution of Coronagraphic Dynamic Range and Constraints on Companions to Vega," Astrophys. J., 654, 633-640 (2007).

[14] Bloemhof, E. E., Troy, M., Dekany, R. G., and Oppenheimer, B. R., "Behavior of remnant speckles in an adaptively corrected imaging system," Astrophys. J. Let., 558, L71-L74 (2001).

[15] Racine, R., Walker, G. A. H., Nadeau, D., Doyon, R., and Marois, C., "Speckle Noise and the Detection of Faint Companions," Publ. Astron. Soc. Pac., 111, 587-594 (1999)

[16] Sparks, W. B. and Ford, H. C., "Imaging Spectroscopy for Extrasolar Planet Detection," Astrophys. J., 578, 543-550 (2002).

[17] Crepp, J.R., Pueyo, L., Brenner, D. et al., "Speckle Suppression with the Project 1640 Integral Field Spectrograph," Astrophys J. , 729, 132-141 (2011).

[18] Pueyo, L., Crepp, J.R., Vasisht, G., et al., "Application of a Damped Locally Optimized Combination of Images Method to the Spectral Characterization of Faint Companions Using an Integral Field Spectrograph," Astrophys. J. Supp. Ser., 199, 6-12 (2011).

[19] Marois, C., Macintosh, B., Barman, T., et al., "Direct Imaging of Multiple Planets Orbiting the Star HR 8799," Science, 322, 1345-1347 (2009).

[20] Oppenheimer, B.R., Brenner, D., Hinkley, S., et al., "The Solar System Scale Disk around AB Aurigae," Astrophys. J., 679, 1574-1582 (2008). Also Hinkley, S., Oppenheimer, B. R., Soummer, R., "Speckle Suppression Through Dual Imaging Polarimetry, and a Ground-based Image of the HR 4796A Circumstellar Disk," Astrophys. J., 701, 804-810 (2009).

[21] Dekany, R., Bouchez, A., Britton, M., Velur, V., Troy, M., Shelton, J.C., and Roberts, J., "PALM-3000: visible light AO on the 5.1-meter Telescope," Proc. SPIE, 6272, (2006). Also, Dekany R., et al. in preparation (2012).

[22] Roberts, J. E., Dekany, R. G., Burruss, R., et al., "Results from PALM-300 high-order adaptive optics system," Proc. SPIE, 8447, 34 (2012).

[23] Soummer, Rémi, "Apodized Pupil Lyot Coronagraphs for Arbitrary Telescope Apertures," Astrophys. J., 618, L61-L65 (2005).

[24] Hinkley, S., Oppenheimer, B. R., Zimmerman, N., Brenner, D., Parry, I. R., Crepp, J. R., Vasisht, G., Ligon, E., King, D., Soummer, R., Sivaramakrishnan, A., Beichman, C., Shao, M., Roberts, L. C., Bouchez, A., Dekany, R., Pueyo, L., Roberts, J. E., Lockhart, T., Zhai, C., Shelton, C. and Burruss, R., "A New High Contrast Imaging Program at Palomar Observatory," Publ. Astron. Soc. Pac., 123, 74-86 (2011).

[25] Wallace, J. K., Burruss, R. S., Bartos, R. D., Trinh, T. Q., Pueyo, L. A., Fregoso, S. F., Angione, J. R., and Shelton, J. C., "The Gemini Planet Imager Calibration Wavefront Sensor Instrument," Proc. SPIE, 7736, $77365 \mathrm{D}(2010)$.

[26] Chengxing, Z. Vasisht, G., Shao, M., et al. "A first-order wavefront estimation algorithm for P1640 calibrator," Proc. SPIE, 8447, 259 (2012).

[27] Zimmerman, N., Brenner, D., Oppenheimer, B.R., Parry, I.R., Hinkley, S., Hunt, S., and Roberts, R., "A Data-Cube Extraction Pipeline for a Coronagraphic Integral Field Spectrograph," Publ. Astron. Soc. Pac., 123, 746-763 (2011).

[28] Oppenheimer, B. R., Sivaramakrishnan, A., and Makidon, R. B., "Imaging Exoplanets: The Role of Small Telescopes," [The Future of Small Telescopes In The New Millennium. Volume III - Science in the Shadows of Giants], Kluwer Academic Publishers, Dordrecht, 155-170 (2003).

[29] Beichman, C. A., Krist, J., Trauger, J. et al., "Imaging Young Giant Planets From Ground and Space," Publ. Astron. Soc. Pac. 122, 62-77 (2010) 
[30] Leconte, J., Soummer, R., Hinkley, S. et al., "The Lyot Project Direct Imaging Survey of Substellar Companions: Statistical Analysis and Information from Nondetections," Astrophys. J., 716, 1551-1570 (2010).

[31] Johnson, J. A., Aller, K. M., Howard, A. W., Crepp, J. S, "Giant Planet Occurrence in the Stellar MassMetallicity Plane," Publ. Astron. Soc. Pac., 122, 905-915 (2010).

[32] Dodson-Robinson, S., Veras, D., Ford, E. B. and Beichman, C. A., "The Formation Mechanism of Gas Giants on Wide Orbits," Astrophys. J., 707, 79-88 (2009).

[33] Marois, C., Zuckerman, B., Konopacky, Q. M., Macintosh, B., Barman, T., "Images of a fourth planet orbiting HR 8799," Nature, 468,1080-1083 (2010).

[34] Lagrange, A. M, Bonnefoy, M., Chauvin, G., et al., "A Giant Planet Imaged in the Disk of the Young Star $\beta$ Pictoris,"2010, Science, 329, 57 (2010).

[35] Baraffe, I., Chabrier, G., Barman, T. S., Allard, F., and Hauschildt, P. H., "Evolutionary Models for Cool Brown Dwarfs and Extrasolar Giant Planets. The case of HD 209458," Astron. \& Astrophys. 435, L13-L17 (2003).

[36] Ibukiyama, A., and Arimoto, N., "HIPPARCOS age-metallicity relation of the solar neighbourhood disc stars," Astron. \& Astrophys., 394, 927-941 (2002).

[37] Zuckerman, B. and Song, I., "Young Stars Near the Sun," Ann. Rev. Astron. Astrophys., 42, 685-721 (2004).

[38] Hillenbrand, L., Carpenter, J. M., Kim, J. S. et al., "The Complete Census of $70 \mu \mathrm{m}$-bright Debris Disks within 'the Formation and Evolution of Planetary Systems' Spitzer Legacy Survey of Sun-like Stars," Astrophys. J., 677, 630-656 (2008).

[39] Holmberg, J., Nordstrom, B. and Anderson, J., "The Geneva-Copenhagen survey of the solar neighbourhood. III. Improved distances, ages, and kinematics," Astron. \& Astrophys., 501, 941-947 (2009).

[40] Vican, L., "Age Determination for 346 Nearby Stars in the Herschel DEBRIS Survey," Astrophys. J., 143, 135 (2012).

[41] Rieke, G. H., Su, K. Y. L., Stansberry, J. A, et al., "Decay of Planetary Debris Disks" Astrophys. J., 620, 1010-1026 (2005).

[42] Crepp, J.R. and Johnson, J.A., "Estimates of the Planet Yield from Ground-based High-contrast Imaging Observations as a Function of Stellar Mass," Astrophys. J., 733, 126-144 (2011).

[43] Nielson, E. and Close, L., "A Uniform Analysis of 118 Stars with High-contrast Imaging: Long-period Extrasolar Giant Planets are Rare Around Sun-like Stars," Astrophys. J., 717, 878-896 (2011).

[44] Roberts, Jr., L. C., Rice, E. L., Beichman, C., et al., "Spectral Typing of Late Type Companions to Young Stars using Near-Infrared Integral Field Unit Data," Astron. J., 144, 14, (2012). 